\section{ADDITIONS TO THE FUNGI OF KERALA - IV}

\section{V.B. Hosagoudar}

Microbiology Division, Tropical Botanic Garden and Research Institute, Palode, Thiruvananthapuram, Kerala 695562, India Email: vbhosagoudar@rediffmail.com

This Note is fourth in a series of additions to the fungi of Kerala published earlier by Hosagoudar et al. (1996) and Hosagoudar (2006a,b,c).

\section{Enumeration of species}

\section{Meliola memecyli Sydow}

On leaves of Memecylon edule, Eanikara, Thiruvananthapuram, 28.x.2000 V.B. Hosagoudar TBGT 662, HCIO 44374; TBGRI campus, Thiruvananthapuram, 10.x.2001, T. Sabu TBGT 659, HCIO 44372; Memecylon sp., Calicut University campus, Kozhikode, 15.v.2001, M. Kamarudeen TBGT 702, HCIO 44373 Nedumangad, Thiruvananthapuram, 5.xii.2001, Mahantesha-gowda, Ishwaragowda \& Basanagowda TBGT 715, HCIO 44375.

Meliola memecyli Sydow var. microspora Hansf.

On leaves of Memecylon gracile, Mannavan shola, Idukki, 19.xi.1999, C.K. Biju TBGT 309, HClO 43618; $M$. edule, near Darsana school, Nedumangad, Thiruvananthapuram, 8.x.2000, P.V. Mahanteshagowda TBGT 1297, HCIO 45259; Vandanam Sacred grove, Alapuzha, 3.x.2002, A. Manojkumar TBGT 982, HCIO 44701

\section{Meliola millettiae-chrysophyllae Deight. var. indica}

Hosag. et al.

On leaves of Derris benthamii, Banasuran mala, Wyanad, 19.xi.1998, C.K. Biju TBGT 310, HCIO 43645.

\section{Meliola mitragynae Sydow}

On leaves of Mitragyna parviflora, Meenmutty, Thiruvananthapuram, 10.iii.1996 V.B. Hosagoudar TBGT 30, HClO 42168

\section{Meliola mitragynae-tubulosae Hosag. et al.}

On leaves of Mitragyna tubulosa, Moozhiyar forest, Pathanamthitta, 14.xii.2004, V.B. Hosagoudar et al. TBGT 1849, HClO 46086

\section{Meliola nairii Hosag.}

On leaves of Aglaia sp., Gavi Area, Pathanamthitta, 6.xii.2004, V.B. Hosagoudar et al. TBGT 1859, $\mathrm{HClO} 46097$.

\section{Meliola neolitseae Yamam.}

On leaves of Neolitsea scrobiculata, Champatty, Silent Valley, 14.xii.2003, V.B. Hosagoudar et al. TBGT 1697, HCIO 45935; Banasuranmala, Wyanad, 19.xi.1999, C.K. Biju HCIO 43463, TBGT 286; Sairandhri, Silent Valley, Palghat, 15.xii.2003, V.B. Hosagoudar et al. TBGT 1674, HClO 45912; Neolitsea sp., Banasuranmala, Wyanad, 16.iv.1999, C.K. Biju TBGT 1206, HCIO 45151 Cryptocarya sp., Chembra hills, Wyanad, 17.ii.2000, C.K. Biju TBGT 341, HCIO 43697.

\section{Meliola nothopegiae Hansf.}

On leaves of Nothopegia aureofulva, Bonacaud, Thiruvananthapuram, 8.iii.2003, G. Rajkumar TBGT 1181, HClO 45126.

\section{Meliola oldenlandiae Hansf. \& Stev.}

On leaves of Hedyotis sp., Mannavan shola, Idukki, 23.ix.1998, C.K. Biju TBGT 233; 21.i.2003, A. Manojkumar \& H. Biju TBGT 1337, HCIO 45176.

Meliola oldenlandiae Hansf. \& Stev. var. indica Hosag. et al. On leaves of Hedyotis sp., Rajamala, Idukki, 25.viii.1998, C.K. Biju TBGT 342 HCIO 43696; Mannavan shola, Munnar, Idukki, 5.i.1999, C.K. Biju TBGT 343 HClO 43695; Gravel Banks, Munnar, Idukki, 20.i.2003, A. Manojkumar \& H. Biju TBGT 1338, HClO 45177; Mannavan shola, Munnar, Idukki, 21.i.2003, A Manojkumar \& H. Biju TBGT 1106, HCIO 44878.

\section{Meliola oleacearum Hosag.}

On leaves of Olea dioica, TBGRI campus, Palode, Thiruvanan-thapuram, 11.xi.2000, H. Biju TBGT 822, HCIO 44536; Kulamavu, Idukki, 6.ix.2001, M. Kamarudeen TBGT 571, HCIO 44187; TBGRI campus, Palode, Thiruvananthapuram, 1.i.2001, H. Biju TBGT 848, HClO 44561; 18.xii.2001, H.
Meliola oleicola Doidge

On leaves of Ligustrum sp., Banasuran mala, Wyanad, 16.iv.1999, C.K. Biju TBGT 1332, HCIO 45294.

Meliola oligomera Sydow

On leaves of Hippocratea sp., Periya, Wyanad, 27.xii.2002, M. Kamarudeen \& P.A. Jose TBGT 1036, HClO 44799.

\section{Meliola otonephelii Hosag.}

On leaves of Otonephelium stipulacearum, Kulamavu, Idukki, 19.v.2001, M.Kamarudeen TBGT 538, HCIO 44151; Tenth mile, Gavi, Pathanamthitta 16.xii.2004, V.B. Hosagoudar et al. TBGT 1804, HCIO 46041; TBGT 1855, HCIO 46092.

Meliola otophorae Yates var. indica Hosag. \& Ravikumar

On leaves of Sapindus laurifolius, Near Valicode, Nedumangad, Thiruvananthapuram, 5.x.2001, Ishwaragowda, Mahantesha-gowda \& Basanagowda TBGT 580; Lepisanthes senegalensis, Chinnar, Idukki, 1.vii.1998, C.K. Biju TBGT 311, HCIO 43644.

\section{Meliola palawanensis Sydow}

On leaves of Morinda umbellata, Vandanam Sacred grove, Alapuzha, 3.x.2002, A. Manoj Kumar TBGT 943, HCIO 44661.

\section{Meliola panici Earle}

On leaves of Brachiaria sp., TBGRI campus, Palode, Thiruvananthapuram, 5.ix.2002, H. Biju \& A. Manojkumar TBGT 1041, HClO 44804; Cymbopogon citratus, Karikulam, Ranni, Pathanamthitta, 4.x.2002, A. Manoj Kumar TBGT 974, HCIO 44693; Cymbopogon sp., Kombe, Meenmutty, Thiruvanantha-puram, 11.iii.1996, V.B. Hosagoudar TBGT 62, HCIO 42192; TBGRI campus, Palode, Thiruvananthapuram, 18.xii.2001, H. Biju TBGT 820, HClO 44534; Sankali forest, Kollam, 23.xi.2004, V.B. Hosagoudar et al. TBGT 1932, HCIO 46286; Member of Poaceae (an unidentified grass), Peppara Wildlife Sanctuary, Thiruvananthapuram, 5.ii.1997, V.B. Hosagoudar TBGT 1161, HCIO 45106; Kombe, Thiruvananthapuram, 19.ii.1997, V.B. Hosagoudar TBGT 1174, HCIO 45119; TBGT 1241, HCIO 45205; TBGRI campus, Thiruvananthapuram, 4.xi.2000, H. Biju TBGT 739, HClO 44450; Karimancode, Palode, Thiruvananthapuram, 21.i.2005, Archana et al. TBGT 1630, HCIO 46218; TBGT 1277, HCIO 45239; Oplismenus compositus, Sankali forest, Kollam, 27.xii.2001, V.B. Hosagoudar TBGT 608, HCIO 44377; 23.xi.2004, V.B. Hosagoudar TBGT 1573, HClO 46161; Panicum sp., Sankali forest, Kollam, 23.xi.2004, V.B. Hosagoudar TBGT 1811, HCIO 46048; TBGT 1610, HClO 46198; 1732, HCIO 45968.

Meliola panici Earle var. vetiveriicola Gawande et al.

On leaves of Vetiveria zizanoides, TBGRI campus, Palode, Thiruvananthapuram, 3.xii.2001, H. Biju TBGT 864, HClO 44577.

\section{Meliola paramignyae Hosag.}

On leaves of Paramignya sp., Sairandhri, Silent Valley, Palghat, 13.xii.2003, V.B. Hosagoudar et al. TBGT 1940, HClO 46294; Champatty, Silent valley, Palghat, 14.xii.2003, V.B. Hosagoudar et al. TBGT 1526, HCIO 45777

\section{Meliola pelliculosa Sydow}

On leaves of Terminalia catappa, Near Eanikara, Thiruvananthapuram, 28.i.2001, P.V. Mahanteshagowda TBGT 397, HClO 43820.

\section{Meliola pepparaensis Hosag. \& Abraham}

On leaves of Tabernaemontana sp., Eanikara, Thiruvanan-thapuram, 9 xii.2000, V.B. Hosagoudar TBGT 441, HClO 43958; TBGRI campus, Palode, Thiruvananthapuram, 18.xii.2001, H. Biju TBGT 870, HCIO 44583; Parakuzhy forest area, Karikulam, Ranni, Pathanamthitta, 4.x.2002, A. Manoj Kumar TBGT 930, HClO 44648; Vazhachal, Trissur, 4.x.2002, H. Biju \& A. Manoj Kumar TBGT 945, HClO 44663.

Meliola pequensis Hosag. et al.

On leaves of Millettia ovalifolia, TBGRI campus, Palode, Thiruvananthapuram, 11.xi.2000, H. Biju TBGT 819, HClO 44533; 5.xii.2001, H. Biju TBGT 760, HCIO 44470; 12.xii.2001, H. Biju TBGT 759, HCIO 44469.

\section{Meliola perseae Stev.}

On leaves of Persea macarantha, Central Nursery, TBGRI campus, Palode, Thiruvananthapuram,15.iv.2005, P.A. Jose TBGT 1923, HClO 46277; Sairandhri, Silent valley, Palghat, 12.xii.2003, V.B. Hosagoudar et al. TBGT 1964, HCIO 46318; Kakki dam, Moozhiyar forest, Pathanamthitta, 15.xii.2004, V.B. Hosagoudar TBGT 1578, HClO 46166; TBGT 1581, HCIO 46169; Sankali forest, Kollam, 23.xi.2004, V.B. Hosagoudar et al. TBGT 1933, HClO 46287; Persea sp., towards Pachakanam, Pathanamthitta, 15.xii.2004, V.B. Hosagoudar et al

(C) Zoo Outreach Organisation; www.zoosprint.org Manuscript 1528; Received 18 February 2006; Revised received 25 May 2006; Finally accepted 09 June 2006 ; Date of publication 21 October 2006 
TBGT 1858, HCIO 46095 .

\section{Meliola phaseoli Thite ex Hosag.}

On leaves of Vigna pilosa, in the forest area near Kushavoor, Palode Thiruvananthapuram, 16.xi.2002, A. Manoj Kumar TBGT 1022, HClO 44785; Placherry forest, Ranni, Pathanamthitta, 6.xii.2002, A. Manoj Kumar TBGT 1051, HClO 44814.

Meliola phyllostachydis Yamam. var. microspora Hosag. et al. On leaves of Bambusa sp., Munnar, Idukki, 20.ix.1998, C.K. Biju TBGT 1265 $\mathrm{HClO}$ 45228; Synarundineria microphylla, Mannavan shola, Munnar, Idukki, 6.i.1999, C.K. Biju TBGT 347, HCIO 43692.

\section{Meliola pogostemonis Hansf.}

On leaves of Pogostemon heyneana, Moozhiyar forest, Pathanamthitta, 14.xii.2004, V.B. Hosagoudar et al. TBGT 1815, HClO 46052; P. pubescens, Bonacaud, Thiruvananthapuram, 13.iii.1997, V.B. Hosagoudar TBGT 195; Pogostemon sp., Rajamala, Idukki, 5.ix.1998, C.K. Biju TBGT 247, HCIO 42968; Mannavan shola, Munnar, Idukki, 2.vi.2000, C.K. Biju TBGT 1138, HCIO 45083 Mattupetty, Munnar, Idukki, 1.i.2003, H. Biju \& A. Manojkumar TBGT 1057 $\mathrm{HClO} 44820$; 1.i.2003, H. Biju \& A. Manojkumar TBGT 1083, HCIO 44846; Vagupara, Munnar, Idukki, 22.i.2003, H. Biju \& A. Manojkumar TBGT 1082, HClO 44845 .

\section{Meliola polytricha Kalch. \& Cooke}

On leaves of Pittosporum neelgherrense, Agastyar Hill, Thiruvananthapuram 26.iii.1996, V.B. Hosagoudar TBGT 102, HCIO 42299.

\section{Meliola pongamiae Hosag. \& Abraham}

On leaves of Pongamia pinnata, Peringamala, Thiruvanantha-puram, 25.xi.2000, M. Kamarudeen TBGT 443, HClO 43965; TBGRI campus, Thiruvananthapuram, 20.xi.2000, V.B. Hosagoudar TBGT 442, 43950; 18.xii.2000, H. Biju HCIO TBGT 828, 44542; 23.i.2001, H. Biju HCIO TBGT 850, 44563; 5.i.2001, H. Biju HCIO TBGT 854, 44567; 13.vi.2001, P.A. Jose TBGT 606; 12.xii.2001, H. Biju HCIO TBGT 783, 44493

\section{Meliola prataparajii Hosag. \& Abraham}

On leaves of Loranthus sp., Peppara Wildlife Sanctuary, Thiruvananthapuram, 28.i.1997, V.B. Hosagoudar TBGT 506, HCIO 44068; TBGRI campus, Thiruvananthapuram, 28.i.2001, Justin P. Jacob TBGT 593, HClO 44382; 8.iii.2001, M. Kamarudeen TBGT 671, HCIO 44380; Eanikara, Thiruvananthapuram, 17.iv.2001, P.V. Mahanteshagowda TBGT 44, HClO 43988; 14.i.2001, V.B. Hosagoudar TBGT 380, HClO 43797; 18.x.2001, V.B. Hosagoudar TBGT 650, HCIO 44381.

Meliola pseudarthriae Hosag. \& Manoj.

On leaves of Pseudarthriae viscida, Sankali forest, Kollam, 23.xi.2004, V.B. Hosagoudar TBGT 1611, HCIO 46199.

\section{Meliola pterocarpi Yates}

On leaves of Pterocarpus marsupium, Peppara Wildlife Sanctuary, Thiruvananthapuram, 3.ii.1997, V.B. Hosagoudar TBGT 144, HClO 42494; 6.ii.1997, V.B. Hosagoudar TBGT 445, HCIO 43951; Kombe, Thiruvananthapuram, 19.ii.1997, V.B. Hosagoudar TBGT 196; TBGRI campus, Thiruvananthapuram, 20.i.2001, V.B. Hosagoudar TBGT 446, HClO 44030 20.i.2001, V.B. Hosagoudar TBGT 447, HClO 44028; 18.xii.2001, H. Biju TBGT 784, HClO 44494; 18.xii.2001, H. Biju TBGT 782, HCIO 44492; 8.iii.2001, H. Biju TBGT 742, HClO 44453

Meliola randiicola Hansf.

On leaves of Ixora sp., Sankali forest, Kollam, 27.xii.2001, V.B. Hosagoudar TBGT 620, HClO 44369.

\section{Meliola rauvolfiae Mibey}

On leaves of Rauvolfia verticillata, Gravel Banks, Munnar, Idukki, 18.xii.2001, S. Shiburaj TBGT 628, HCIO 44383.

\section{Meliola rubiella Hansf.}

On leaves of Rubus sp., Rajamala hills, Idukki,5.ix.1998, C.K. Biju TBGT 227, HClO 42931; Wagamon, Kottayam, 5.xii.2002, A. Manoj Kumar TBGT 1006, $\mathrm{HClO} 44725$.

\section{Meliola sairandhriana Hosag.}

On leaves of Aglaia minutiflora, Sairandhri, Silent valley, Palghat, 14.xii.2003 V.B. Hosagoudar et al. TBGT 1902, HClO 46139; Aglaia sp., Champatty, Silent Valley, Palghat, 14.xii.2003, V.B. Hosagoudar et al. TBGT 1989, HCIO 46343.
Meliola samaderae Hosag. et al.

On leaves of Quasia indica, TBGRI campus, Thiruvanantha-puram, 12.xii.2000, S. Shiburaj TBGT 448, HCIO 43975; 20.i.2001, S. Shiburaj TBGT 507, HCIO 44055; 5.i.2001, H. Biju TBGT 797, HCIO 44511; 2.iii.2005, G.R. Archana \& V.L. Sheeja TBGT 1690, HCIO 45928.

\section{Meliola scleropyri Hosag.}

On leaves of Scleropyrum pentandrum, Attayar, Thiruvananthapuram, 19.iii.1997, V.B. Hosagoudar TBGT 125, HClO 42484.

\section{Meliola semecarpi-anacardii Hosag. et al.}

On leaves of Semecarpus anacardium, Peppara Wildlife Sanctuary, Thiruvananthapuram, 5.ii.1997, V.B. Hosagoudar TBGT 145, HClO 42495 Semecarpus sp., Ponmudi, Thiruvananthapuram, 7.iii.2003, G. Rajkumar \& P.A. Jose TBGT 1165, HCIO 45110.

Meliola serjaniae Stev. var. major. Hansf.

On leaves of Sapindus laurifolius, TBGRI campus, Palode, Thiruvananthapuram, 18.i.2001, H. Biju TBGT 823, HClO 44537.

Meliola simillima Ellis \& Ever. var. major Hansf.

On leaves of Holarrhena antidysenterica, in the campus of Museum, Thiruvananthapuram, 7.i.2003, G. Rajkumar TBGT 1346, HCIO 45185.

\section{Meliola spatholobi Hosag. et al.}

On leaves of Spatholobus parviflorus, TBGRI campus, Palode, Thiruvananthapuram, 23.i.2001, H. Biju TBGT 824, HCIO 44538; S. roxburghii, Sankali forest, Kollam, 23.xi.2004, V.B. Hosagoudar et al. TBGT 1892, HCIO 46129; TBGT 1894, HCIO 46131; TBGT 1917, HCIO 46271; Vankulla, Sankali, Kollam, 23.xi.2004, V.B. Hosagoudar et al. TBGT 1795, HCIO 46032; Spatholobus sp., Shendurney, Kollam, 14.i.2003, V.B. Hosagoudar et al. TBGT 1135, HCIO 45080 .

\section{Meliola spigeliae Hansf.}

On leaves of Strychnos nux-vomica, Cheppad, Alappuzha, 23.xi.2000, M. Kamarudeen TBGT 365, HCIO 43822; TBGRI campus, near DBT building, Palode, Thiruvananthapuram, 18.i.2001, H. Biju TBGT 860, HCIO 44573; TBGRI campus, Palode, Thiruvananthapuram, 23.i.2001, H. Biju TBGT 821, HCIO 44535; Pullumudy, Chalakudy, Trissur, 22.x.2002, H. Biju \& A. Manoj Kumar TBGT 987 $\mathrm{HClO} 44706$.

\section{Meliola stenospora Wint.}

On leaves of Piper sp., Wyanad, 5.ii.2002, M. Kamarudeen TBGT 610, HCIO 44385.

Meliola stenospora Wint. var. major Hansf.,

On leaves of Piper nigrum, Kulamavu, Idukki, 26.iv.2003, G. Rajkumar \& P.A Jose TBGT 1211, $\mathrm{HClO} 45156$

\section{Meliola strebili Hosag.}

On leaves of Streblus taxoides, near Sasthanamada tribal colony, Sankali forest, Kollam, 23.xi.2004, V.B. Hosagoudar TBGT 1605, HClO 46193; S. asper, Sankali forest, Kollam, 23.xi.2004, V.B. Hosagoudar et al. TBGT 1889, $\mathrm{HClO}$ 46126; Streblus sp., Tribal colony, Sasthanamada, Sankali forest, Kollam, 23.xi.2004, V.B. Hosagoudar et al. TBGT 1893, HCIO 46130.

\section{Meliola strobilanthicola Hosag. et al.}

On leaves of Strobilanthes sp., Nalpathanchushola, Munnar, Idukki, 22.i.2003, H. Biju \& A. Manojkumar TBGT 1055, HClO 44818; Vagupara, Munnar Idukki, 22.i.2003, H. Biju \& A. Manojkumar TBGT 1075, HClO 44838.

Meliola suttonii Hosag. et al.

On leaves of Litsea sp., Gravel banks, Munnar, Idukki, 18.xii.2001, S. Shiburaj TBGT 1229, HCIO 45189.

\section{Meliola syzygii-benthamiani Hosag. \& Abraham}

On leaves of Syzygium benthamianum, Athirumala, Neyyar Wildlife Sanctuary, Thiruvananthapuram, 26.iii.1996, V.B. Hosagoudar TBGT 1235, HCIO 45199.

\section{Meliola tabernaemontanae Speg.}

On leaves of Tabernaemontana sp., Rajamala, Idukki, 5.ix.1998, C.K. Biju TBGT 248, HCIO 42966; Mannavan shola, Munnar, Idukki, 5.i.1999, C.K.Biju TBGT 344, HClO 43694.

Meliola tabernaemontanicola Hansf. \& Thirum.

On leaves of Tabernaemontana gamblei, Devikulam, Munnar, Idukki, 23.i.2003, A. Manojkumar \& H. Biju TBGT 1339, HClO 45178; T. heyneana, Vagupara, Munnar, Idukki, 22.i.2003, A. Manoj Kumar \& H. Biju TBGT 1284, HCIO 45246; 
Nalpathanchushola, Munnar, Idukki, 1.i.2003, A. Manoj Kumar \& H. Biju TBGT 1287, HClO 45249; Tabernaemontana sp., Vaguparai, Munnar, Idukki, 2.i.2003, A. Manoj Kumar \& H. Biju TBGT 1136, HClO 45081; Vallaris solanacea, in the Museum campus, Thiruvananthapuram, 28.xii.2002, G. Rajkumar TBGT 1120, $\mathrm{HClO}$ 44892; TBGRI medicinal plant garden, Palode, Thiruvananthapuram, 5.i.2001, H. Biju TBGT 867, HCIO 44580.

\section{Meliola tamarindi Sydow}

On leaves of Tamarindus indicus, Chathanoor, Kollam, 10.xii.1997, C.K.Biju TBGT 449, HClO 44005; Eanikara, Thiruvananthapuram, 1.i.2001, V.B. Hosagoudar TBGT 450, HClO 44029; Placherry forest, Ranni, Pathanamthitta, 4.x.2002, A. Manoj Kumar TBGT 933, HCIO 44651.

Meliola tecleae Hansf. var. toddaliae-asiaticae Hansf.

On leaves of Toddalia asiatica, Tirunelly, Wyanad, 11.viii.1998, C.K. Biju TBGT 249, HClO 42963; Mannavan shola, Idukki, 6.i.1999, C.K. Biju TBGT 283, $\mathrm{HClO} 43446$.

\section{Meliola telosmae Rehm var. bogeriensis Hansf}

On leaves of Asclepiadaceae member, Rajamala, Idukki, 7.i.1999, C.K. Biju TBGT 314, HCIO 43641; Gymnema sylvestre, TBGRI campus, Thiruvananthapuram, 10.xii.2002, M. Kamarudeen TBGT 989, HCIO 44708; TBGRI Nursery, Palode, Thiruvananthapuram, 6.i.2003, P.A. Jose TBGT 1053, $\mathrm{HClO} 44816$.

\section{Meliola tenella Pat.}

On leaves of Murraya paniculata, TBGRI campus, Palode, 25.i.2005, G.R. Archana \& V.L. Sheeja TBGT 1807, HClO 46044; Moozhiyar forest, Pathanamthitta, 14.xii.2004, V.B. Hosagoudar et al. TBGT 1859, HClO 46096; Palode, Thiruvananthapuram, Naranayan Nair TBGT 522, HCIO 44120; TBGRI campus, Thiruvananthapuram, 12.x.2001, M.M. Shajivaz TBGT 704, HCIO 44387.

\section{Meliola tenella Pat. var. atalantiae (Pat.) Hansf.}

On leaves of Atalantia monophylla, Chinnar, Idukki, 1.vii.1998, C.K. Biju TBGT 205, $\mathrm{HClO} 42885$.

\section{Meliola teramni Sydow}

On leaves of Teramnus labialis, Moozhiyar forest, Pathanamthitta, 14.xii.2004, V.B. Hosagoudar et al. TBGT 1797, HClO 46034

\section{Meliola tetradeniae Berk.}

On leaves of Neolitsea sp., Athirumala, Neyyar Wildlife Sanctuary Thiruvananthapuram, 10.iii.1996, TBGT 228, HCIO 42932; TBGT 345.

\section{Meliola themediicola Hosag. et al.}

On leaves of Themeda triandra, Chembra hills, Wyanad, 17.ii.2000, C.K. Biju TBGT 315, HClO 43640.

\section{Meliola tibigirica Hosag. \& Abraham}

On leaves of Rhynchospora corymbosa, Kombe, Thiruvanan-thapuram, 19.ii.1997, V.B. Hosagoudar TBGT 521, HCIO 44121

\section{Meliola toddaliae Doidge}

On leaves of Pambrus missionis, 4.xii.1996, V.B.Hosagoudar TBGT 105, HCIO 42432; TBGRI campus, Thiruvananthapuram, 26.viii.1998, K. Anilkumar TBGT 451, HCIO 43976; 9.i.2001, M. Kamarudeen TBGT 508, HClO 44063; 17.i.2001, H. Biju TBGT 827, HClO 44541; 5.viii.2002, Mathew Dan TBGT 802, HCIO 44516; 4.xi.2000, H. Biju TBGT 794, HClO 44508; 12.xii.2001, H. Biju TBGT $758, \mathrm{HClO} 44468$.

Meliola toddalicola Doidge var. indica Hasnf. \& Thirum.

On leaves of Toddalia sp., Devikulam, Munnar, Idukki, 23.i.2003, A. Manojkumar \& H. Biju TBGT 1249, HClO 45212.

\section{Meliola toonae Hosag. \& Sabu}

On leaves of Melia dubia, Central nursery, TBGRI campus, 22.iii.2005, P.A Jose TBGT 1735, HCIO 45971; Toona ciliata, TBGRI arboretum, Palode, Thiruvananthapuram, 1.ix.2002, H. Biju TBGT 882, HCIO 44595.

\section{Meliola toreniae Hosag.}

On leaves of Torenia malabarica, Gravel banks, Rajamala, Munnar, Idukki, 20.i.2003, H. Biju \& A. Manojkumar TBGT 1068, HCIO 44831; T. travancorica, 18.x.2001, S. Shiburaj TBGT 711, HCIO 44388; 18.xii.2001, S. Shiburaj TBGT 632, HClO 44389; Torenia sp., Champatty, Silent valley, Palghat, 14.xii.2003, V.B. Hosagoudar et al. TBGT 1718, HCIO 45954; TBGT 1720, HCIO 45956.

\section{Meliola unonicola Hosag. \& Abraham}

On leaves of Meiogyne pannosa (Miliusa pannosa), Banasuran mala, Wyanad, 16.iv.1999, C.K.Biju TBGT 317, HCIO 43638; TBGT 382, HCIO 43832; Chemunji,
Thiruvananthapuram, 3.i.2001, G. Rajkumar TBGT 1090, HCIO 44862.

\section{Meliola wendlandiae Hosag.}

On leaves of Wendlandia notoniana, TBGRI campus, 12.xii.1997, V.B Hosagoudar TBGT 146, HCIO 42496; W. thyrsoidea, Chembra hills, Wyanad, 11.xi.1998, C.K. Biju TBGT 319, HCIO 43635; TBGT 346, HClO 43693 Brahmagiri, Wyanad, 6.xi.2001, S.Shiburaj TBGT 714, HCIO 44390; Sairandhri, Silent Valley, Palghat, 13.xii.2003, V.B. Hosagoudar et al. TBGT 1554, HCIO 45805; Rubiaceae member, Brahmagiri, Tirunelli, Wyanad, 17.vii.2002, S. Shiburaj TBGT 808, HCIO 44522.

Meliola zanthoxyli Hansf.

On leaves of Zanthoxylum tetraspermum, Mannavan shola, Munnar, Wyanad, 2.vi.2000, C.K.Biju TBGT 326, HCIO 43634.

\section{Meliola ziziphi Hansf. \& Thirum.}

On leaves of Ziziphus jujuba, Placherry forest, Ranni, Pathanamthitta, 4.x.2002, A. Manoj Kumar TBGT 927, HClO 44645; Z. oenoplea, Kombe, Meenmutty, 9.iii.1996, V.B. Hosagoudar TBGT 33, HClO 42171; Sankali forest, Kollam, 23.xi.2004, V.B. Hosagoudar TBGT 1615, HCIO 46203; Z. rugosus, Kombe, Meenmutty, 11.iii.1996, V.B. Hosagoudar TBGT 65, HCIO 42195; 19.ii.1997, V.B. Hosagoudar TBGT 198; Peppara Wildlife Sanctuary, Thiruvananthapuram, 4.ii.1997, V.B. Hosagoudar TBGT 181, HClO 42506; Z. trinervia, Champatty, Silent valley, Palghat, 14.xii.2003, V.B. Hosagoudar et al. TBGT 1719, HCIO 45955; Ziziphus sp., Champatty, Silent Valley, Palghat, 14.xii.2003, V.B. Hosagoudar et al. TBGT 1699; forest area, Kusavoor, Palode, Thiruvananthapuram, 16.xi.2002, A. Manojkumar TBGT 968, HClO 44687; Banasuran mala, Wyanad, 16.iv.1999, C.K. Biju TBGT 327, HCIO 43633.

\section{Meliola sp.}

On leaves of Litsea sp., Sairandhri, Silent valley, 12.xii.2003, V.B. Hosagoudar et al. HClO 46327, TBGT 1973; Plectronia sp., Mannavan shola, Munnar, Idukki, 21.i.2003, A. Manojkumar \& H. Biju TBGT 1171, HCIO 45116.

\section{Meliolina pulcherrima (Sydow \& Sydow) Sydow \& Sydow}

On leaves of Syzygium cumini, TBGRI campus, Thiruvanan-thapuram, 5.vii.2001, M.M. Shajivaz TBGT 676, HCIO 44391; Achankovil, Kollam, 8.i.2003, M. Navas TBGT 1025, HClO 44788; Periya, Wyanad, 21.iv.2003, G. Rajkumar \& P.A. Jose TBGT 1124, HCIO 44895; Nallathani, Murinjapuzha, Peerumedu, Idukki, 25.viii.2003, M. Navas TBGT 1130, HClO 45075; Syzygium sp., Munnar, Idukki, May 2003, G. Bagyanarayana TBGT 1756, HClO 45992.

\section{Microxyphium sp.}

On leaves of Gordonia sp., Munnar, Idukki, 29.viii.2002, S. Shiburaj TBGT 1959, HClO 46313; Actinodaphne sp., Gavi, Pathanamthitta, 16.xii.2004, V.B. Hosagoudar TBGT 1664, HClO 46252.

\section{Monodyctis sp.}

On leaves of Glycosmis sp., Tirunelly, Wyanad, 20.v.2002, S. Shiburaj TBGT 978, HClO 44697.

\section{Myriostigmella sp.}

On leaves of Psychotria flavida, Meenmutty, Peppara and Neyyar Wildlife Sanctuaries, Thiruvananthapuram, 10.iii.1996, V.B. Hosagoudar TBGT 2032.

\section{Oidium abutili Hosag.}

On leaves of Abutilon sp., Kakki dam, Moozhiyar forest, Pathanamthitta, 14.xii.2004, V.B. Hosagoudar TBGT 1941, HClO 46295.

Oidium doidgeae Bapp. et al.

On leaves of Triumfetta sp., Sairandhri, Silent Valley, Palghat, 15.xii.2003, V.B. Hosagoudar et al. TBGT 1675, HCIO 45913.

\section{Oidium hemidesmi Singh \& Kamal}

On leaves of Hemidesmus indicus, Peppara Wildlife Sanctuary, Thiruvananthapuram, 6.ii.1997, V.B. Hosagoudar TBGT 534, HCIO 44134.

\section{Oidium heveae Stein.}

On leaves of Hevea sp., Kombe, Meenmutty, Thiruvananthapuram, 18.ii.1997, V.B. Hosagoudar TBGT 481, HCIO 43985.

\section{Oidium indigoferae Yen}

On leaves of Indigofera tinctoria, Njakkadu, Varkala, Kollam, 8.ii.1997, S. Shiburaj TBGT 182.

Oidium peltophori (Yen) Boes. var. indica Hosag. et al. On leaves of Peltophorum pterocarpum, Peppara Wildlife Sanctuary, Thiruvananthapuram, 3.ii.1997, V.B. Hosagoudar 452. 


\section{Oidium ramakrishnanii Hosag.}

On leaves of Phyllanthus sp., in the campus of KSEB rest house, Moozhiyar forest, Pathanamathitta, 14.xii.2004, V.B. Hosagoudar et al. TBGT 1749, HCIO 45985; near Anathode dam area, Moozhiyar forest, Pathanamthitta, 15.xii.2004, V.B. Hosagoudar et al. TBGT 1673, HClO 45911.

Oidium seaforthiani Hosag et al.

On leaves of Solanum seaforthianum, Bonacaud, Thiruvananthapuram, 13.iii.1997, V.B. Hosagoudar TBGT 189

Oidium tamarindi (Yen) Braun

On leaves of Tamarindus sp., Kombe, Thiruvananthapuram, 18.ii.1997, V.B. Hosagoudar TBGT 535, HClO 44137

Oidium ziziphi (Yen \& Wang) Braun

On leaves of Ziziphus sp., Moozhiyar forest, Pathanamthitta, 14.xii.2004, V.B. Hosagoudar \& al TBGT 1845, HClO 46082.

Olivea tectonae (Ramakr. \& Ramakr.) Mueller

On leaves of Tectona grandis, Sri Nilayam, Nedumangad, Thiruvananthapuram, 28.xii.2001, V.B. Hosagoudar TBGT 600, HCIO 44393; Nedumangad, Thiruvananthapuram, 12.xii.2001, V.B. Hosagoudar TBGT 630, HCIO 44392.

\section{Ophiodothella calamicola (Ramakr. \& Ramakr.) Hyde \& Cannon}

On leaves of Calamus sp., Attayar, Thiruvananthapuram, 8.iii.1997, V.B. Hosagoudar TBGT 453, $\mathrm{HClO} 43994$; on the way to Kulathupuzha, Kollam, 13.i.2005, V.B. Hosagoudar et al. TBGT 1710, HCIO 45946; TBGT 1764, HCIO 46000 .

\section{Ophiodothella lagerstroemiae Hosag. \& Nair}

On leaves of Lagerstroemia microcarpa, Peppara Wildlife Sanctuary, Thiruvananthapuram, 6.ii.1997, V.B. Hosagoudar TBGT 457; Anapaddy, MPCA Parambikulam Reserve forest, Palghat, 25.xi.2003, K. Ravikumar \& al TBGT 1500, HClO 45751; near Sairandhri, Silent Valley, Palghat, 15.xii.2003, V.B. Hosagoudar et al. TBGT 1711, HCIO 45947; Uppupara, Pachakanam, Pathanamthitta, 16.xii.2004, V.B. Hosagoudar TBGT 1669, HClO 46257; Moozhiyar forest. Pathanamathitta, 14.xii.2004, V.B. Hosagoudar et al. TBGT 1705, HClO 45941; Lagerstroemia sp., Peppara Wildlife Sanctuary, Thiruvananthapuram, 4.ii.1997, V.B. Hosagoudar TBGT 454, HCIO 43962; TBGT 456, $\mathrm{HClO}$ 43018; Attayar, Thiruvananthapuram, 20.iii.1997, V.B. Hosagoudar TBGT $455, \mathrm{HClO} 44021$

\section{Passalora pongamiae (H. Syd.) Deight.}

On leaves of Pongamia pinnata, Neyyar dam, Thiruvanan-thapuram, 17.ii.1997, V.B.Hosagoudar TBGT 712, $\mathrm{HClO} 44394$

\section{Periconiella persae-macranthae Hosag. \& Braun}

On leaves of Persea macrantha, Peppara Wildlife Sanctuary, Thiruvananthapuram, 3.ii.1997, V.B. Hosagoudar TBGT 458, HCIO 44022; TBGRI campus, Thiruvananthapuram, 20.xii.2000, P.A. Jose TBGT 459, HCIO 44026; Shendurney Wildlife Sanctuary, Kollam, 14.i.2003, V.B. Hosagoudar TBGT 37 HClO 44850; Konni, Pathanamthitta, 4.xii.2002, A. Manoj Kumar TBGT 1002, $\mathrm{HClO} 44721$.

\section{Phaeoxyphiella sp.}

On leaves of Trema orientalis, Kushavoor, Palode, Thiruvananthapuram, 11.i.2003, A. Manojkumar TBGT 1958, HClO 46312.

\section{Phakopsora apoda (Pat. \& Har.) Mains stat. Uredinia}

On leaves of Pennisetum polystachyon, Kombe, Meenmutty, Thiruvananthapuram, 11.iii.1996, V.B. Hosagoudar TBGT 66, HCIO 42196.

Phleospora cassiae Thirum. \& Narasim.

On leaves of Cassia fistula, Sankali, Kollam, 23.xi.2004, V.B. Hosagoudar TBGT 1703, HCIO 45939; TBGT 1721, HCIO 45957.

Pheoseptoria thespesiae Ramakr.

On leaves of Thespesia populnea, Near railway station, Tellicherry, 17.xi.2003, V.B. Hosagoudar TBGT 1626, HCIO 46214

\section{Phyllachora abri (Subhedar \& Rao) Cannon}

On leaves of Abrus precatorius, Kombe, Meenmutty, Thiruvananthapuram, 9.iii.1996, V.B. Hosagoudar TBGT 106, HCIO 42433

Phyllachora ambigua (Sydow) Sydow

On leaves of Syzygium cumini, Karimancode, Palode, Thiruvananthapuram, 21.i.2005, Archana et al. TBGT 1635, HCIO 46223.
Phyllachora catarvaria (Berk.) Sacc.

On leaves of Ficus sp., Gavi, Pathanamthitta, 16.xii.2004, V.B. Hosagoudar TBGT 1666, HClO 46254; Moozhiyar forest, KSEB quarters, Pathanamathitta, 14.xii.2004, V.B. Hosagoudar et al. TBGT 1729, HCIO 45965.

Phyllachora cynodontis (Sacc.) Niessl.

On leaves of Cynodon dactylon, TBGRI Arboretum, Palode, 12.ix.2002, K. Vijayakumar TBGT 1347, HClO 45186.

\section{Phyllachora ischaemi Sydow}

On leaves of Cymbopogon sp, Athirumala, Neyyar Wildlife Sanctuary, 26.iii.1996, V.B. Hosagoudar TBGT 67, HCIO 42197

Phyllachora microcenta (Berk. \& Br.) Sacc.

On leaves of Artocarpus hirsutus, Sankali forest, Kollam, 5.i.2001, H. Biju TBGT 804, HClO 44518.

\section{Phyllachora millettiae Henn.}

On leaves of Millettia rubiginosa, Ponmudy, Thiruvananthapuram, 24.i.2005, V.B. Hosagoudar et al. TBGT 1708, HCIO 45944; Karadimala, Neyyar Wildlife Sanctuary, 8.iii.1996, V.B. Hosagoudar TBGT 107, HCIO 42434.

\section{Phyllachora pongamiae (Berk. \& Br.) Petch}

On leaves of Pongamia pinnata, TBGRI campus, Thiruvananthapuram, 5.ii.2002, V.B. Hosagoudar TBGT 592, HClO 44395; 3.i.2002, H. Biju TBGT 611, HCIO 44396; Museum, Thiruvananthapuram, 9.ix.2000, Mrs. Sharada TBGT 647, HCIO 44399.

Phyllachora sikkimensis Ramkr., T.S.

On leaves of Canarium strictum, Athirumala, Neyyar Wildlife Sanctuary, 25.iii.1996, V.B. Hosagoudar TBGT 108, HClO 42435; Central Nursery, TBGR campus, Thiruvananthapuram, 9.xi.2001, P.A. Jose TBGT 687, HClO 44397.

\section{REFERENCES}

Hosagoudar, V.B. (2006a). Additions to the fungi of Kerala. Zoos Print Journal 21(7): 2322-2330.

Hosagoudar, V.B. (2006b). Additions to the fungi of Kerala - II. Zoos Print Journal 21(9): 2412-2416.

Hosagoudar, V.B. (2006c). Additions to the fungi of Kerala - III. Zoos Print Journal 21(10): 2421-2424.

Hosagoudar, V.B., T.K. Abraham and P. Pushpangadan (1996) Fungi of Kerala. Tropical Botanic Garden and Research Institute, Palode, Thiruvananthapuram, $151 \mathrm{pp}$.

\section{ACKNOWLEDGEMENTS}

Thanks are due to Dr. S. Ganeshan, Director, TBGRI, Palode for the facilities. I thank all the persons who have contributed the collections to TBGT micro fungal herbarium. I am grateful to Mr. B.V. Shetty, Emeritus Scientist, Mangalore University, Karnataka for reviewing the manuscript critically.

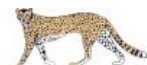

